\title{
Hiding Multi Short Audio Signals in Color Image by using Fast Fourier Transform
}

\author{
Enas M. Jamel* \\ Computer Science Department, College of Education for Women-University of Baghdad, Baghdad, Iraq
}

\begin{tabular}{ll}
\hline \multicolumn{1}{c}{ Article's Information } & \multicolumn{1}{c}{ Abstract } \\
\hline Received: & Many purposes require communicating audio files between the users using \\
19.09 .2020 & different applications of social media. The security level of these applications is \\
Accepted: & limited; at the same time many audio files are secured and must be accessed by \\
14.02 .2021 & authorized persons only, while, most present works attempt to hide single audio \\
Published: & file in certain cover media. In this paper, a new approach of hiding three audio \\
13.03 .2021 & signals with unequal sizes in single color digital image has been proposed using \\
\hline Keywords: & the frequencies transform of this image. In the proposed approach, the Fast \\
Steganography & Fourier Transform was adopted where each audio signal is embedded in specific \\
Secret audio signal & region with high frequencies in the frequency spectrum of the cover image to \\
Security & save much more details of the cover image and avoid any doubts that there is \\
Image frequency transform & any secret information are hidden inside it. The quality of the stego-image and \\
Hiding multi audio & the extracted audio files are evaluated with the standard evaluation metric. The \\
Image quality & simulation results shown significant results of these metrics and achieve good \\
& imperceptibility and high security of the stego-image. The SNR and SPCC \\
& values are considered acceptance that means significant in terms quality and \\
\hline
\end{tabular}

DOI: 10.22401/ANJS.24.1.09

*Corresponding author: enasm.altai@gmail.com

\section{Introduction}

During the past ten years, the process of exchanging information between the users using different social media applications takes biggest attention around the world. Some of this information is secret, and only authorized peoples are accepted to access the true content. This issue can be addressed easily by encrypting this information using specific standard technique. Another way for this issue is by using steganography technique. This technique hides the secret information in another cover media called stego object which can be video, image, and audio, but the digital image is more popular media as a stego object according to its large scale to hide more information. Also, the secret information can be text message, video, audio, and image [1,2]. In general, there are three main characteristics of any steganography system. The first is imperceptibility which stands for the ability of this system to not be recognized by the human beings. The second is the capacity of secret information which is measured in bits/bytes/samples. The third is security which measures the percent of difficulty to detect the secret information; also it can be used to obtain the quality of the secured signal [3].

Mainly, there are two steganography techniques. The first technique implements steganography in temporal or spatial domain, where the secret information are embedded directly in the actual samples of the stego object $[4,5]$.
While, in the second technique the stego object is transformed form spatial domain to another domain using Fast Fourier Transform (FFT), Discrete Cosine Transform (DCT) or Discrete Wavelet Transform (DWT), and then the secret information are embedded in determined coefficients of this transform.

Some types of attacks can be verified against the stego object like (noise, shifting, scaling, etc.). The stego object resulted by temporal domain has significant reliability for updating than transform domain against any kind of attacks that affected this object. The actual samples of this object are sensitive for updating than the transformed coefficient, thus, the transform domain is more robustness than the temporal against different types of attacks [5].

The Fourier transform is a method used for converting from time domain to the frequency domain, and it is called discrete Fourier transform, or DFT, in the case of digital signals. It is useful when converting multi-dimensional data from the spatial domain to the frequency domain. So it can be used in many different operations such as data compression, spread spectrum and watermarking. In the field of digital signal processing, transforms are widely used in analyzing and processing discrete data and commonly used computational mathematics [6]. The Image is two-dimensional quantities, the Fourier transform of an image is represented as two-dimensional. The Fourier transform of an image is usually represented by the 


\title{
Al-Nahrain Journal of Science
}

\author{
ANJS, Vol.24 (1), March, 2021, pp. 57-65
}

equivalent form known as magnitude and phase. The Fourier transform is a complex function that can be represented as real and imaginary parts of a complex function. The real part corresponds to the terms of cosine, and the imaginary part corresponds to the terms of sine. magnitude and phase are a representation of a polar coordinate transformation in which each sine/sine pair of corresponding magnitude and phase is specified [7]. FFT is considered to be as one of the straightest and most efficient methods for converting to and from the frequency domain [6].

The proposed steganography approach aims to hide multi audio signals within single color image, implemented method by using the Discrete Fourier Transform, The algorithm for computing the DFT is very fast, known as Fast Fourier Transform. Both the stegoimage and reconstructed audio signals evaluated by using the standard evaluation metrics to prove the effectiveness of the proposed approach to perform steganography issue.

This paper is organized as follows. Section 2 presented the related work. Section 3 presented the Discrete Fourier Transform (DFT). Section 4 presented Verification measurements. Section 5 describes the proposed method including data embedding, extraction. Experimental results and performance comparisons with other result of DWT, are shown in Section 6. Finally, conclusions are given in Section 7.

\section{Related Work}

As the main goal of the steganography technique is to hide secret information in another media. Many articles found in literature deals with hiding (text, images, and videos) in digital image, but very few approaches are proposed to embed audio signal in digital image [8]. In [9], a steganography technique has been proposed to hide audio signal (MP3 or WAV) in color image using DWT. The audio signal is compressed before hiding using DCT. The Experimental results show good values for evaluation metrics. Another technique was proposed by [8] to embed short audio message in the least significant bit of the stego digital image using wavelet transform. In this technique, the cover image is converted from RGB to $\mathrm{YCbCr}$ color mode, and then both the audio and image are transformed using integer wavelet transform. The embedding scenario is to implement $\mathrm{x}$-or operation for each 2 bits from the approximation coefficient of audio signal with 2 bits from the $\mathrm{Cb}$ coefficient and then store the results in the corresponding 2 bits of $\mathrm{Cr}$ coefficients. The simulation results achieved the similarity and quality between original and stego image. In paper [10], describes Image Steganography the secret audio file is hidden in the image by applying DWT (Haar Wavelet) on both cover image and audio signal, any format of audio file is embedded in $\mathrm{HH}$ and HL sub-band of cover image. There are no clear changes on the cover image after embedding secret data. In [11], this paper is focused on hidden secret audio signal a color image by using the AES algorithm and circular LSB algorithm. The audio is embedded by both AES algorithm with LSB algorithm, in arrangement of data into LSB bit of pixel of image. Password is used for encrypted image for more security in communication.

Fast Fourier Transform (FFT) is a transform method, which converts the signal into the frequency domain. Several papers have been published with this transform method in steganography technique. In this paper [12], the technique of steganography is used FFT to hide a two secret image into the Fourier magnitude of the cover image that is by replacing the high-frequency with the data of the hidden images. In paper [6], apply FFT on video frames to convert the cover to frequency domain, then hide the encrypt logo image in cover frames by using least two significant bit technique. The result shows, this method does not have any effected on the quality of original video after inserting logo image in it. In paper [13], presents to efficient way of watermarking based on DWT and FFT, implemented 2 decomposition DWT on cover image, then LL2 is divided in to $8 * 8$ blocks since apply FFT on the block is selected to embed the watermark image. This paper denotes a fast approach and new proposed algorithm does not appear any deteriorate on the cover image. In paper [14], discusses the method of embedded secret image into audio. Apply FFT on cover audio to convert it to the frequency domain. The secret image is compressed by wavelet compression technique, then convert it to binary form. When hiding secret image in the audio, the amplitude of cover audio must be modified by using a secret key. Secret image can be extracted from stego audio effectively. In paper [15], the cover audio is decomposed by means of using the Lifting Wavelet Transform (LWT) to obtain several sub-bands, then FFT used for selected lowest energy sub-bands. Watermark image is converted into one-dimensional signal, then it is modulated. The experimental result of this method has good imperceptibility. In [16], the paper describes secure speech communication by applying DWT and FFT. DWT is used to separate high-frequency components of the lowfrequency components of the speech. And then embedded secret speech signal in the high-frequency regions low amplitude of the cover speech signal, that achieve by using the low-pass spectral proprieties of the speech spectrum. This method permits aimed at embedding a large amount of secret information and the secret speech message can be recovered it with a small degradation in the quality.

\section{Discrete Fourier Transform (DFT)}

The discrete Fourier transform is a completely discrete transform that can be converted discrete time signals into a discrete number of frequencies. DFT is used to convert a finite list that has equally spaced samples of a function into the list of coefficients of a finite combination of complex sinusoids ordered by their frequencies. It can be said the sampled function has been converted from its original domain, that can be time or position along a line in the frequency domain. [2]. This algorithm is known as the Fast 


\title{
Al-Nahrain Journal of Science
}

\author{
ANJS, Vol.24 (1), March, 2021, pp. 57-65
}

Fourier Transform (FFT). Let $f(x, y)$ be the spatial value of a matrix with size $N \times N$. The frequency domain transformation is performed by the following formula:

$$
F(u, v)=1 / N^{2} \sum_{x=0}^{N-1} \sum_{y=0}^{N-1} f(x, y) e^{\left(-j 2 \pi\left(\frac{u x}{N}+\frac{v y}{N}\right)\right)}
$$

indicate to the two-dimensional (2D) Fourier transform of an image $f(x, y)$, the size of $N$ by $\mathrm{N}$ where $x=0,1,2, \ldots, N$ -1 and $y=0,1,2, \ldots, N-1$, and $j$ is assumed to be present the square root of -1 . The complex exponential is denoted at this point as a summation of cosines and sines with different frequencies [7, 12].

As well, the spatial representation field is produced from the frequency representation when applied Inverse Fast Fourier Transform (IFFT) on it. The following equation describes IFFT.

$$
f(x, y)=1 / N^{2} \sum_{u=0}^{N-1} \sum_{v=0}^{N-1} F(u, v) e^{\left(-j 2 \pi\left(\frac{u x}{N}+\frac{v y}{N}\right)\right)}
$$

where $u=0$ to $N-1, v=0$ to $N-1$.

The FFT is considered to be as one of the straightest and most efficient methods for converting to and from the frequency domain [6].

\section{Verification Measurements}

\section{(a) Evaluation metrics of the cover image}

The process of embedding specific information inside the media of image causes some degradation to this media. The strength of any steganography approach reduces this degradation and makes the stego-image seems to be similar to the original image before embedding. This similarity and the quality of the stego-image is measured using some standard metrics which are considered by wellknown similar works.

The first metric is Mean Squared Error (MSE) which is used to measure the difference between two images. This metric is obtained using the definition mentioned in equation (3). A small value of the MSE metric means high similarity level between the stego-image and original image. The second metric is Peak Signal-to Noise Ratio (PSNR) which is used to measure the quality of the stegoimage. This metric is obtained using the definition mentioned in equation (4). Unlike MSE, a high value of PSNR means significant quality of the stego-image [4]. Typically, the assortment of PSNR values between 20 - 40 to good quality image [16].

$$
\begin{aligned}
& M S E=\frac{1}{m \times n} \sum_{i=0}^{m-1} \sum_{j=0}^{n-1}[O(i, j)-S(i, j)]^{2} \\
& P S N R=10 . \log _{10}\left(\frac{\max ^{2}}{M S E}\right)
\end{aligned}
$$

where $m$ and $n$ are the width and height of the image; $O(i, j), S(i, j)$ are the original and stego-image respectively.

Another metric, called Structural Similarity Index for measuring (SSIM), which is used to obtain the variation of brightness, contrast, and structure of the image. The SSIM is obtained using the definition mentioned in equation (5) and its result improves the MSE and PSNR values [17].

$$
S S I M=\frac{\left(2 . \mu_{x} \cdot \mu_{y}+C_{1}\right)\left(2 . \sigma_{x y}+C_{2}\right)}{\left(\mu_{x}^{2}+\mu_{y}^{2}+C_{1}\right)\left(\sigma_{x}^{2}+\sigma_{y}^{2}+C_{2}\right)}
$$

where $\mu_{\mathrm{x}}$ is the average value of all pixels in first image, and $\sigma_{x}$ is the square average difference between $\mu_{x}$ and each pixel in the image and the same thing is for $\mu_{y}, \sigma_{y}$ in the second image. The $\sigma_{x y}$ is the average difference between $\sigma_{x}$ and $\sigma_{y}$. The $\mathrm{C} 1$ and $\mathrm{C} 2$ are two constant which is used to avoid zero value in the denominator. The calculations of these contestants are performed by equation (6) and (7), respectively.

$$
\begin{aligned}
& C_{1}=\left(W_{1} P\right) \\
& C_{2}=\left(W_{2} P\right)
\end{aligned}
$$

where $P$ is the maximum pixel value in the image (usually 255 for 8 bits/pixel), and $W_{1}$ and $W_{2}$ are fixed with very low values. In $[5,10], W_{1}$ is set to $(0.01)$ and $W_{2}$ is set to (0.03). The effective range of SSIM is limited between $(-1$ and 1). The fully identical images give maximum SSIM value equal to 1 .

(b) Evaluation metrics of the reconstructed signals There are two evaluation metrics which are considered to evaluate the quality of the reconstructed signals. The first metric is signal to noise ratio (SNR) which is used to measure the percent level of any signal with respect to the noise existing inside this signal. The measurement unit of SNR is decibels (dB). The high value of SNR means significant quality of the reconstructed signal because the level of signal exceeds the level of noise existing. This metric is mostly used for quantity evaluation but cannot be considered for quality evaluation [8]. The SNR is obtained using the definition mentioned in equation (8)

$$
\mathrm{SNR}=10 \cdot \log _{10}\left(\frac{\frac{1}{\mathrm{~N}} \sum_{\mathrm{i}=0}^{\mathrm{N}} \mathrm{x}_{\mathrm{i}}^{2}}{\mathrm{MSE}}\right)
$$

The second evaluation metric for reconstructed signal is Squared Pearson Correlation Coefficient (SPCC) which is used to measure the similarity level of the two signals. The high value for this metric reflects the significant level of similarity between the two signals [19]. The SPCC is obtained using the definition mentioned in equation (9).

$$
\operatorname{SPCC}=\left[\frac{\sum_{i=1}^{N}\left(x_{i}-\mu_{x}\right)\left(y_{i}-\mu_{y}\right)}{\sqrt{\sum_{i=1}^{N}\left(x_{i}-\mu_{x}\right)^{2}} \sqrt{\sum_{i=1}^{N}\left(y_{i}-\mu_{y}\right)^{2}}}\right]^{2}
$$

where $\left(x_{i}, y_{i}\right)$ are the original and the reconstructed signals. The $\left(\mu_{x}, \mu_{y}\right)$ are the average value of all pixels in the original and the reconstructed signals, respectively.

\section{Proposed Steganography Approach}

As mentioned previously, the proposed steganography approach aims to hide multi audio signals inside single color image. Any format for the audio files (mp3, wav, etc.) can be used in the proposed approach. Also, any format for the color (png, bmp, jpg, etc.) can be considered for the stego-image. Since, any audio file with few seconds duration using various formats contains hundreds thousands of samples, the size of audio files used (not exceed 256,000 samples). Thus, the stego-image must be large enough to handle this huge information of audio samples. The embedding process steps shown in Figure 1. 


\section{Al-Nahrain Journal of Science}

ANJS, Vol.24 (1), March, 2021, pp. 57-65

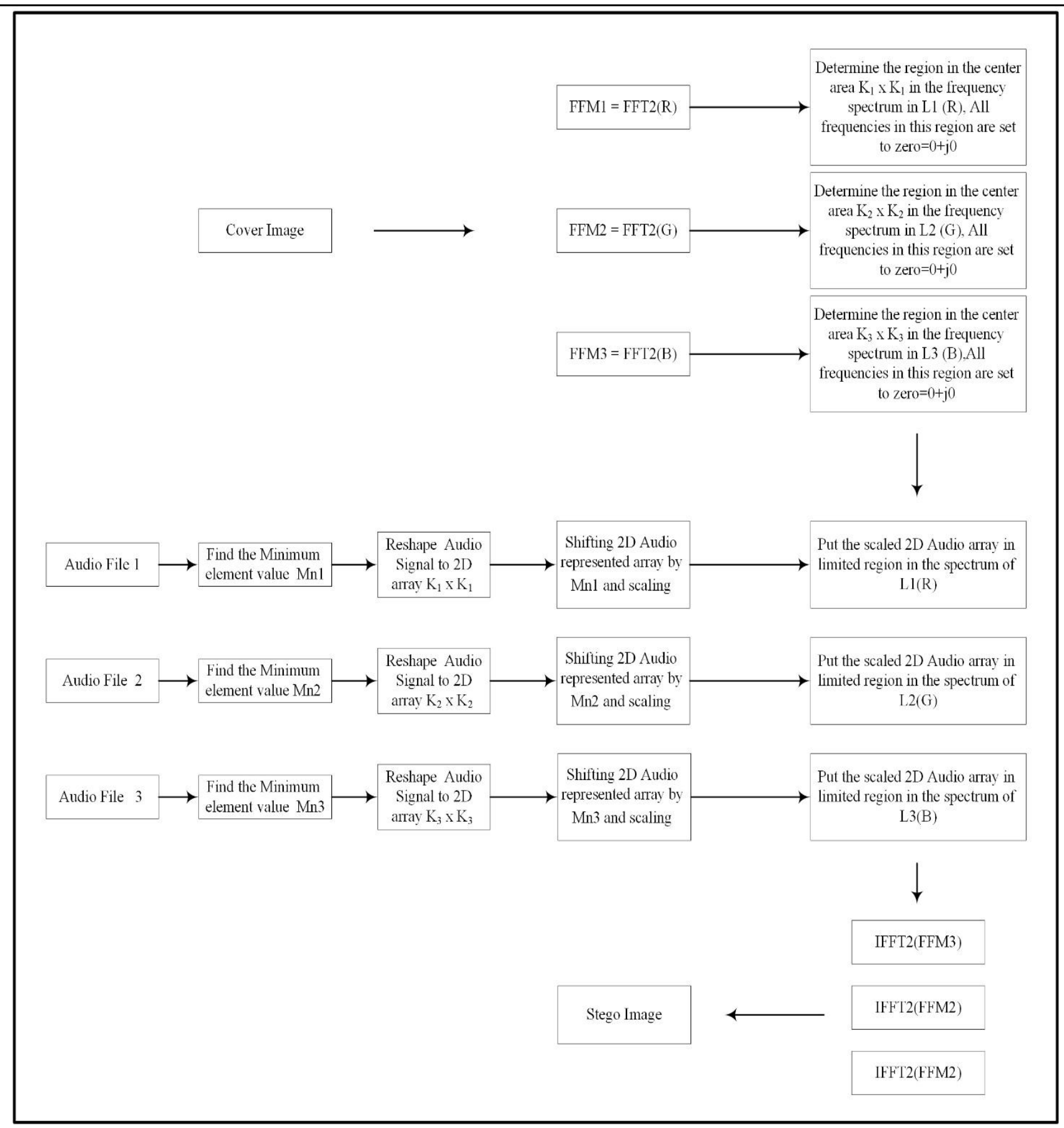

Figure 1. The block diagram of the embedding process.

The steps of the proposed steganography approach are mentioned in Embedding Algorithm. 


\title{
Al-Nahrain Journal of Science
}

ANJS, Vol.24 (1), March, 2021, pp. 57-65

\begin{abstract}
Proposed Algorithm of Embedding Three Audio Signals in Single Color Image
BEGIN

[L1, AUD1]=Read Audio File(<Audio File Name 1>) ／* read 1st Audio file of length L1 */

[L2, AUD2]=Read Audio File (<Audio File Name 2>) $\quad / *$ read 1st Audio file of length L2*/

[L3, AUD3]=Read Audio File (<Audio File Name 3>) $\quad / *$ read 1st Audio file of length L3*/

Mn1=Min Value(AUD1) $\quad / *$ Find the Minimum element value in $1^{\text {st }}$ audio signal $* /$

Mn2=Min Value(AUD2) $\quad / *$ Find the Minimum element value in $2^{\text {nd }}$ audio signal $* /$

Mn3=Min Value(AUD3) $\quad / *$ Find the Minimum element value in $3^{\text {rd }}$ audio signal $* /$
\end{abstract}

[Wd, Ht, IMG]=Read Color Image $(<$ FILENAME $>) \quad / *$ read color image in RGB format with $\mathrm{Wd}$ width and $\mathrm{Ht}$ height*/

[Wa1, Ha1, Arr_A1=Reshape to 2Darray ( AUD1[1,..,L1]) /* Reshape 1st Audio Signal to $2 \mathrm{D}$ array with Wa1 rows and Ha1 Columns */

[Wa2, Ha2, Arr_A2=Reshape to 2Darray( AUD2[1,..,L2]) $\quad / *$ Reshape $2^{\text {nd }}$ Audio Signal to $2 \mathrm{D}$ array with $\mathrm{Wa} 2$ rows and $\mathrm{Ha} 2$ Columns */

[Wa3, Ha3, Arr_A3=Reshape to 2Darray( AUD3[1,..,L3]) $\quad / *$ Reshape $3^{\text {rd }}$ Audio Signal to $2 \mathrm{D}$ array with $\mathrm{Wa} 3$ rows and Ha3 Columns */
$\operatorname{Nor}_{\mathrm{ADD} 1}=(\mathrm{Mxx} *($ Arr_A1-Mn1) $\quad / *$ Shifting 2D Audio represented array by Mn1 and scaling result with high thousand value e.g $3000 * /$
Nor $_{\mathrm{ADD} 2}=(\mathrm{Mxx} *($ Arr_A2-Mn2) $) \quad / *$ Shifting 2D Audio represented array by Mn2 and scaling result with high thousand value $e . g 3000 * /$
Nor $_{\mathrm{ADD} 3}=(\mathrm{Mxx} *($ Arr_A3-Mn3) $) \quad / *$ Shifting 2D Audio represented array by Mn3 and scaling result with high thousand value e.g $3000 * /$

FFM=FFT2(IMG) $\quad / *$ Take 2D FFT for the Color Image */

$/^{*}$ replace the region in the center area in the frequency spectrum in layer $\mathrm{L}: 1, . .3$ which is limited by $(\mathrm{Ht} / 4, .$. $\mathrm{Ht} / 4+\mathrm{HaAn}(\mathrm{An}))$ horizontally and $(\mathrm{Wd} / 4, . ., \mathrm{Wd} / 4+\mathrm{WaAn})$ vertically for auth An:1,..,3 Audio Signals */

REAL(FFM(L) $\left.\left.\left[\mathrm{Ht} / 4, . ., \mathrm{Ht} / 4+\mathrm{Ha}_{\mathrm{An}}(\mathrm{An}), \mathrm{Wd} / 4, . ., \mathrm{Wd} / 4+\mathrm{Wa}_{\mathrm{An}}\right)\right]\right)=\operatorname{Nor}_{\mathrm{ADD}(\mathrm{An})}$

IMAGINARY(FFM(L) $\left.\left.\left[\mathrm{Ht} / 4, . ., \mathrm{Ht} / 4+\mathrm{Ha}_{\mathrm{An}}(\mathrm{An}), \mathrm{Wd} / 4, . ., \mathrm{Wd} / 4+\mathrm{Wa}_{\mathrm{An}}\right)\right]\right)=$ zero $\quad{ }^{*}$ setting the imaginary part of the limited region to zero */

$\mathrm{IMG}_{\mathrm{Stego}}=\operatorname{IFFT} 2(\operatorname{REAL}(\mathrm{FFM}(\mathrm{L})+\mathrm{j}$ IMAGINARY $(\mathrm{FFM}(\mathrm{L})) \quad / *$ take the inverse $2 \mathrm{D}$ IFFT2 of the resulted three frequency spectrums of $\operatorname{FFM}(\mathrm{L}), \mathrm{L}: 1, . .3$ to generate Stego-image $\mathrm{IMG}_{\text {Stego }} *$ /

END.

The first step in the proposed methodology is to take two dimensional FFT (2-D FFT) for the color image. As the color image has three color layers (RGB), three frequency spectrums are generated. These spectrums are with the same size of the color image and according the basic concepts of FFT as a compound series of sine and cosine functions, the frequency spectrum are with complex elements [12]. The absolute or amplitude value for one frequency spectrum is mentioned in Figure 2.

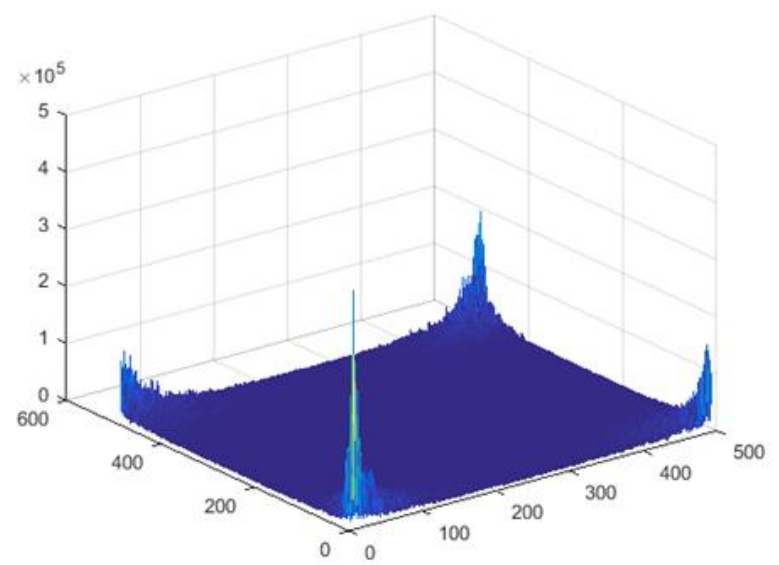

Figure 2. The absolute spectrum of one layer in color image. 


\section{Al-Nahrain Journal of Science}

ANJS, Vol.24 (1), March, 2021, pp. 57-65

As shown in Figure 2, the absolute frequency elements in the corner region of the spectrum takes very high amplitude compared to the elements towards the center region. This distribution comes from the basic definition of FFT including the balance difference between the real and imaginary part of each element, so the real part represented by the cosine function of angle zero in the corner takes maximum value equal to one while the imaginary part represented by the sine value takes zero value for the same angle. This scenario will gradually have inversed towards the center region. From the previous, the frequency elements in the corner region which represent low frequencies take very high amplitudes, while the other elements towards the center region which represent high frequencies take small amplitude. According the basic concepts of frequency elements distribution in the frequency spectrum of the FFT [7, 12], any updating applied on the low frequency elements lead to hard effects on the original media after applying inverse transform, while this effect will be much less for the high frequency elements. The second step in proposed steganography approach based on this truth by replacing limited area from the center region with the audio information. The audio information is arranged in one dimensional set and their absolute amplitude is low (less than one) as shown in the audio sample signal mentioned in Figure 3. Thus before embedding operation, all samples of secret audio signal must be reshaped to two dimensional array and scaled up to match the nature of center region in frequency spectrum.

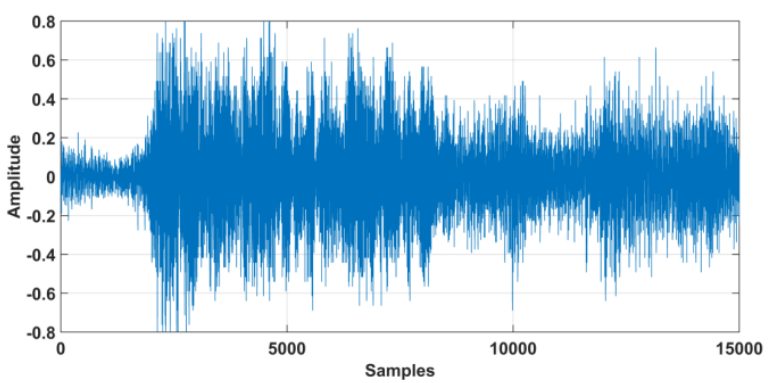

Figure 3. Sample of audio signal.

In the third step in the proposed approach, the two dimensional inverse Fourier transform is applied on the resulted frequency spectrum from the previous step after replacing limited center region the scaled audio information. The result from this step is the stego-image that hides the audio information. The steps of the proposed steganography approach are mentioned in Algorithm.

In the receiving side, two steps are followed to reconstruct the secret audio signals which are embedded within the stego-color image $\mathrm{IMG}_{\text {Steg. }}$. The first step is to take 2-D FFT for the stego-color image. Now, the audio signals are extracted from the three frequency spectrums which are resulted from the inverse transform. Only the dimension of the three audio arrays and the scaling parameters must know by the receiver to perform the extraction operation successfully. Finally, each of the three extracted audio array is reshaped again to generate one dimensional audio signal. The Extracting process steps shown in Figure 4.

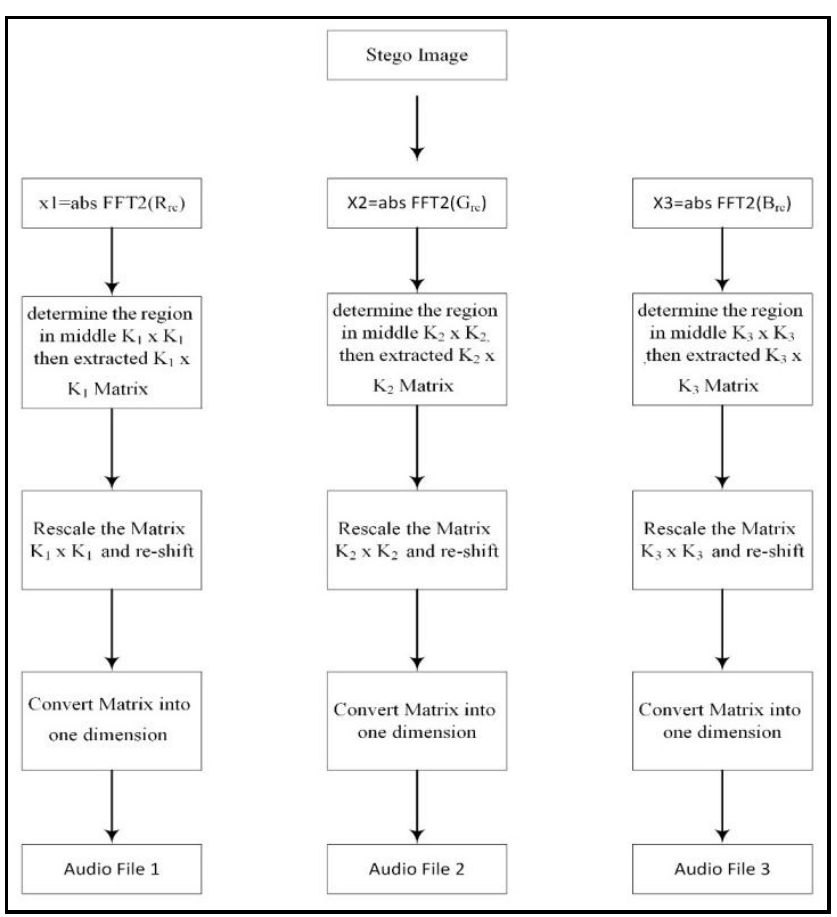

Figure 4. The block diagram of the extracting process.

\section{Experimental Results}

In this section, the analytic performance the proposed steganography approach of embedding multi audio signals within single color image is evaluated qualitatively based on the physical view of image and audio signals, also quantitatively using the standard evaluation metrics for stego and secret information mentioned in section 4. Three audio signals with $(146432,171008,136192)$ samples are considered for validation performance, and the color image used as the stego-object is Lena image of size $(512 \times 512)$. The stego-image and the three reconstructed audio signals can be evaluated qualitatively with the original image and audio signals by visual inspection. The three original audio signals and the reconstructed audio signals after applying the proposed steganography approach are mentioned in Figure 5(a), (b), (c) and Figure 5 (d), (e), (f), respectively. Also, the original image used and the stego-version of this image after embedding three audio signals are mentioned in Figure 6 (a), (b), respectively. 


\section{Al-Nahrain Journal of Science}

ANJS, Vol.24 (1), March, 2021, pp. 57-65
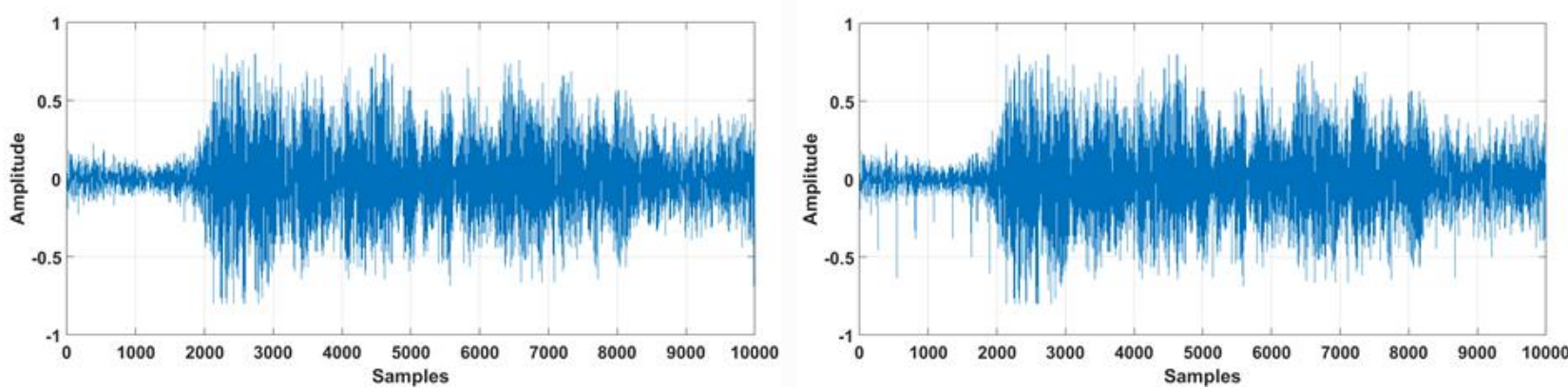

(a)

(d)
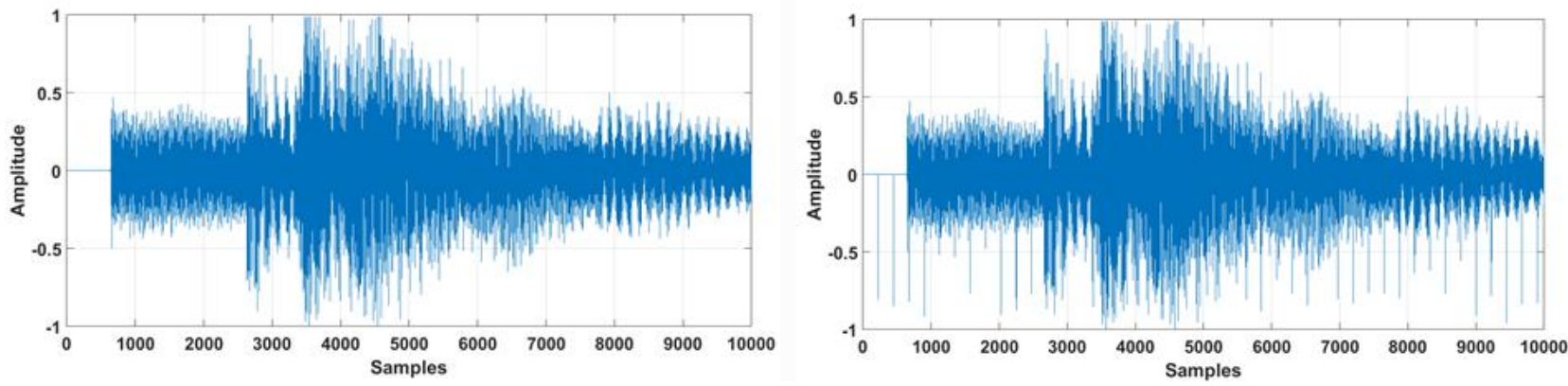

(b)

(e)
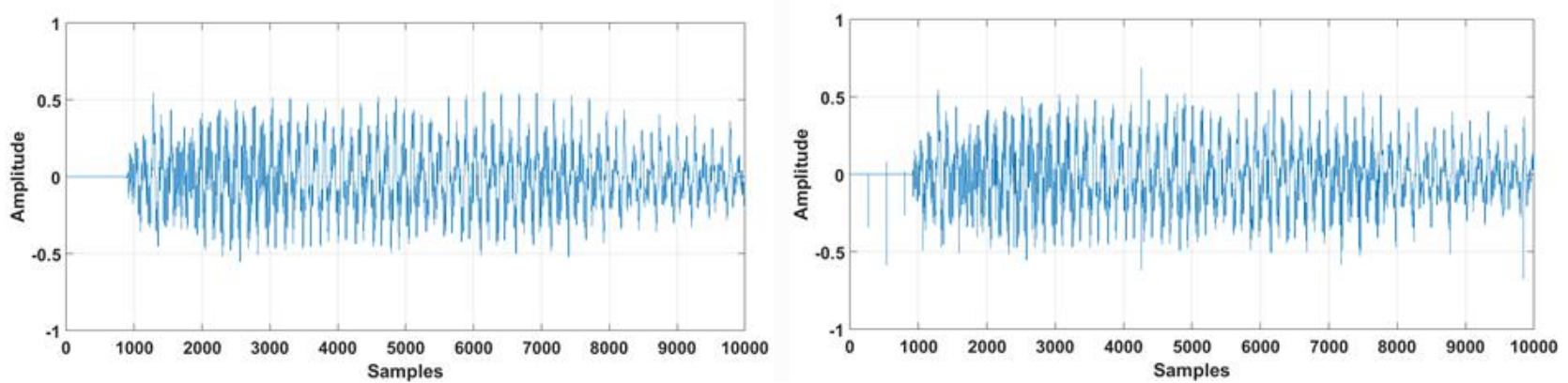

(c)

(f)

Figure 5. The proposed steganography approach: (a), (b), (c) $1^{\text {st }}, 2^{\text {nd }}$ and $3^{\text {rd }}$ : Original audio signals, (d), (e), (f) $1^{\text {st }}, 2^{\text {nd }}$ and $3^{\text {rd }}$ : Reconstructed audio signals.
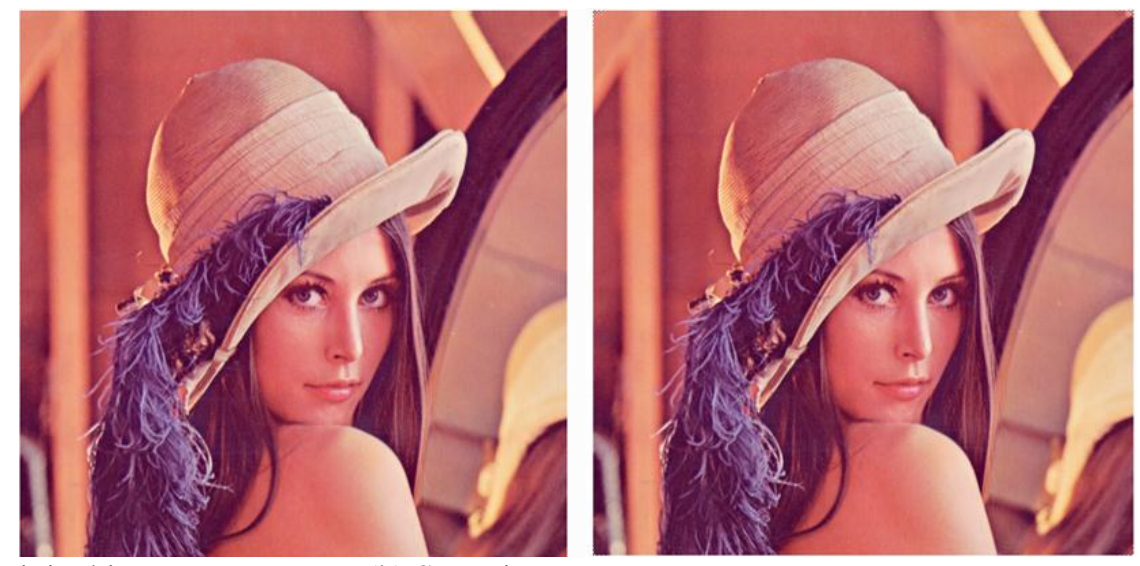

(a) Original image

(b) Stego image

Figure 6. The proposed steganography approach (a): The original Lena image, (b): The stego Lena image.

In Figures 5 and 6, the significant degree of similarity between the original and the reconstructed audio signals using the proposed steganography approach prove the effectiveness of this approach to same much more quality of the audio signal after embedding it inside the color image. Also, the highest percent of similarity between the original and the stego-color image which prevents suspicion of having hidden information inside it and gives 


\title{
Al-Nahrain Journal of Science
}

\author{
ANJS, Vol.24 (1), March, 2021, pp. 57-65
}

the proposed approach high level of security even that three audio signals are embedded inside this image.

Another scenario can be applied to evaluate the performance of the proposed steganography approach quantitatively. In this evaluation, the PSNR and SSIM metrics are considered for stego-image, while for audio signals the SNR and SPCC are considered as the evaluation metrics. The simulation results of these metrics which are obtained by applying the proposed steganography approach on three audio signals with three different numbers of samples $(32768,65536$ and 131064) are illustrated in Table 1 . These results are validated with the corresponding results which are recorded by the existing wavelet transform based steganography technique [8] using the same set of audio samples and same color image.

The validation results mentioned in Table 1 view that the proposed approach scores better results for all quantitative evaluation metrics used for all numbers of audio samples used in the simulation. Also, the simulation results for the three audio signals using same number of samples are very close which prove the balance in distribution secret audio information within the stegoimage.

Table 1. Quantitative evaluation metrics obtained by the proposed steganography approach and other existing technique.

\begin{tabular}{|c|c|c|c|c|c|c|c|}
\hline \multirow{2}{*}{ Method } & \multirow{2}{*}{$\begin{array}{l}\text { Cover } \\
\text { Image }\end{array}$} & \multirow{2}{*}{\multicolumn{2}{|c|}{$\begin{array}{l}\text { No. of Audio Signals } x \\
\text { No. of Secret Samples }\end{array}$}} & \multicolumn{2}{|c|}{ Stego-Image } & \multicolumn{2}{|c|}{ Extracted Audio Signal } \\
\hline & & & & $\operatorname{PSNR}(\mathrm{dB})$ & SSIM & SNR & SPCC \\
\hline \multirow{3}{*}{$\begin{array}{c}\text { wavelet transform based } \\
\text { steganography technique } \\
{[5]}\end{array}$} & \multirow{12}{*}{$\begin{array}{l}\text { LENA } \\
\text { Image } \\
512 \times 512\end{array}$} & \multicolumn{2}{|c|}{$1 \times 32768$} & 41.6 & 0.954 & 38.3 & 0.9022 \\
\hline & & \multicolumn{2}{|c|}{$1 \times 65536$} & 38.6 & 0.935 & 36.3 & 0.8922 \\
\hline & & \multicolumn{2}{|c|}{$1 \times 131064$} & 38.7 & 0.935 & 32.4 & 0.8353 \\
\hline \multirow{9}{*}{$\begin{array}{c}\text { Proposed Steganography } \\
\text { Approach }\end{array}$} & & \multirow{3}{*}{$3 \times 32768$} & $\begin{array}{c}1^{\text {st }} \\
\text { Signal }\end{array}$ & \multirow{3}{*}{45.6} & \multirow{3}{*}{0.9989} & 42.3 & 0.9711 \\
\hline & & & $\begin{array}{c}2^{\text {nd }} \\
\text { Signal }\end{array}$ & & & 41.9 & 0.9585 \\
\hline & & & $\begin{array}{c}3^{\text {rd }} \\
\text { Signal }\end{array}$ & & & 42.1 & 0.9601 \\
\hline & & \multirow{3}{*}{$3 \times 65536$} & $\begin{array}{c}1^{\text {st }} \\
\text { Signal }\end{array}$ & \multirow{3}{*}{42.2} & \multirow{3}{*}{0.9973} & 41.6 & 0.9311 \\
\hline & & & $\begin{array}{c}2^{\text {nd }} \\
\text { Signal }\end{array}$ & & & 40.2 & 0.9136 \\
\hline & & & $\begin{array}{c}3^{\text {rd }} \\
\text { Signal }\end{array}$ & & & 41.3 & 0.9225 \\
\hline & & \multirow{3}{*}{$3 \times 131064$} & $\begin{array}{c}1^{\text {st }} \\
\text { Signal }\end{array}$ & \multirow{3}{*}{41.3} & \multirow{3}{*}{0.9989} & 38.2 & 0.8937 \\
\hline & & & $\begin{array}{c}2^{\text {nd }} \\
\text { Signal }\end{array}$ & & & 37.6 & 0.8885 \\
\hline & & & $\begin{array}{c}3^{\text {rd }} \\
\text { Signal } \\
\end{array}$ & & & 38.1 & 0.8905 \\
\hline
\end{tabular}

\section{Conclusions}

In this study, a new approach of embedding multi audio signals inside single color image has been proposed. In contrast to the existing technique, three different secret audio signals can be hide in the stego-image. The hiding operation of the audio signal is performed by replacing limited region of image frequency spectrum by the secret audio information after scaling all samples of this audio signal to match the amplitude range of the image frequency spectrum. The frequency region of replacing is fixed in the high frequency band to minimize the distortion in the smooth information of the image in low frequency band. Qualitative and quantitative evaluations scenarios are considered to evaluate the performance of the proposed steganography approach. The simulation results view the capability of the proposed approach to reconstruct all the embedded audio signals with high quality and minimum percent of distortion.

\section{References}

[1] Shelke F. M.; Dongre A. A. and Soni P. D.; "Comparison of Different Techniques for Steganography in Images", IJAIEM, 3, 171-176, 2014. IJAIEM-2014-02-27-062.

[2] Kaur H. and Rani J.; "A Survey on different techniques of steganography", MATEC Web of Conferences 57, 02003 ICAET, 2016. DOI: 10.1051/matecconf/ 20165702003.

[3] Abbasi M.; "Color Image Steganography using Dual Wavelet Transforms", International Journal of Computer Applications.181, 32-38, 2019. DOI: 10.5120/ijca2019918639 


\section{Al-Nahrain Journal of Science}

ANJS, Vol.24 (1), March, 2021, pp. 57-65

[4] Hussain M.; Wahab A.W.A.; Idris Y.I.B.; Ho A.T.S. and Jung K. H.; "Image Steganography in Spatial Domain: A survey", Signal Process. Image Commun. 65, 46-66, 2018. DOI:10.1016/j.image.2018.03.012.

[5] Cheddad A.; Condell J.; Curran K. and Kevitt P. M.; "Enhancing Steganography in Digital Images", Canadian Conference on Computer and Robot Vision, 326-332, 2008. DOI: 10.1109/CRV.2008.54. Source: IEEE Xplore.

[6] Hussein A. A. and Al-Thahab O. Q. J.; "Design and Simulation a Video Steganography System by using FFT-Turbo Code Methods for Copyrights Application", Eastern-European Journal of Enterprise Technologies, 104, 43-55, 2020. DOI: 10.15587/17294061.2020.201010.

[7] Rzeszotarski M. S.; Royer F. L. and Gilmore G. C.; "Introduction to Two-Dimensional Fourier Analysis", Behav. Res. Methods Instrumm 15, 308-318, 1983. DOI: $10.3758 / \mathrm{BF} 03203566$.

[8] Hemalatha S.; Acharyaa U. D. and Renuka A.; "Wavelet Transform Based Steganography Technique to Hide Audio Signals in Image", Procedia Comput. Sci., 47, 272-281, 2015. DOI: 10.1016/j.procs.2015.03. 207.

[9] Shah T. A. M.; Talekar A. and Raut P.; "Steganographic Technique for Hiding Secret Audio in an Image", IJREAM 3, 1-6, 2017. IJREAMV03I022640

[10] Shinde A. S. and Patankar A. B.,"Image Steganography: Hiding Audio Signal in Image Using Discrete Wavelet Transform", International Conference On Emanations in Modern Technology and Engineering (ICEMTE), 5, 331-334, 2017. www.ijritcc.org.

[11] Aagarsana B. G.; Anjali, Kirthika T. K. and Sivakumar, S., "Image Steganography using Secured Force Algorithm for Hiding Audio Signal into Colour Image", International Research Journal of Engineering and Technology (IRJET), 5, 1224-1228, 2018. www.irjet.net.

[12] Rabie T.; "Digital Image Steganography: An FFt Approach", Conference in Communications in Computer and Information Science-Springer, 217230,2012. DOI: 10.1007/978-3-642-30567-2_18.

[13] Barua B. and Abedin Z., "An Algorithm for Image Watermarking Based on Discrete Wavelet and Fast Fourier Transform", Proceedings of the 5th International Conference on Natural Sciences and Technology (ICNST’18), 119-123, 2018. http://ilabaustralia.org.

[14] E N. T. and Ravi R. V.; "An Effective Technique for Hiding Image in Audio", International Journal of Science and Research (IJSR), 4, 2984-2987, 2015. www.ijsr.net, ID: SUB156077.

[15] Budiman G.; Suksmono A. B. and Danudirdjo D., "FFT-Based Data Hiding on Audio in LWTDomain Using Spread Spectrum Technique",
ELEKTRONIKA IR ELEKTROTECHNIKA, 26, 2027, 2020. DOI.org/10.5755/j01.eie.26.3.23950.

[16] Rekik S.; Guerchi D.; Selouani S. A. and Hamam H., "Speech steganography using wavelet and Fourier transforms", EURASIP Journal on Audio, Speech, and Music Processing.1-14, 2012. DOI:10.1186/ 1687-4722-2012-20.

[17] Salomon D.; "Data Compression", The complete reference, Fourth Edition; Springer-Verlag London Limited, 2007.

[18] Wang Z.; Bovik A. C.; Sheikh H. R.; Simoncelli E. P.; "Image Quality Assessment: From Error Visibility to Structural Similarity", IEEE T Image process 13, 600-612, 2004. DOI: 10.1109/TIP.2003.819861.

[19] Yan D.; Wang R.; Yu X. and Zhu, J.; "Steganography for MP3 Audio by Exploiting the Rule of Window Switching", Comput. Secur. 31, 704-716, 2012. DOI:10.1016/j.cose.2012.04.006. 